\title{
The role of CD10 immunohistochemistry in the grading of phyllodes tumour of the breast
}

\begin{abstract}
Objective: To determine the relationship between the degree of CD10 expression in the stromal cells of phyllodes tumour and tumour grading. Methods: A total of 61 cases of mammary phyllodes tumours over the past 11 years were searched from histopathology files, University Malaya Medical Centre. The paraffin blocks were retrieved and $4 \mu \mathrm{m}$ thick slides were prepared and stained using an antibody against CD10 with the envision method. Fibroadenoma case was used as a control slide and breast myoepithelium as the internal control. Each stained slides was independantly and semiquantitatively analysed for the intensity and percentage of the stromal cells stained. The staining intensity was graded as negative (no staining), mild, moderate and strong if the staining was much weaker, slightly weaker and same intensity as that of the myoepithelium, respectively. The tumour was considered positive for CD10 if the staining intensity is moderate to strong in $20 \%$ or more of the stromal cells. Results: $21(44.7 \%)$ of 47 benign phyllodes tumour, $5(83.3 \%)$ of 6 borderline phyllodes tumour and all 8 cases $(100 \%)$ of malignant phyllodes tumour showed positive expression for CD10 immunostain. Conclusions: There was a significant increase in CD10 expression in the stromal cells as the lesions progressed from benign to borderline and malignant phyllodes tumour.
\end{abstract}

Keyword: CD10; Phyllodes tumour 